\title{
Présentation de soi sur une application de rencontre - le cas des hommes gays en mobilité étudiante
}

\section{Self-presentation on a dating application - the case of gay men studying abroad}

\author{
Nicolas Guichon \\ Université du Québec à Montréal (UQÀM), CRIFPE, Département de didactique des langues
}

\begin{abstract}
Résumé. Située dans le champ de recherche des études sur le numérique et la migration, cette étude explore de quelles façons les étudiants étrangers gays se présentent sur une application de rencontre (Grindr) et se forgent une identité intermédiaire entre leur pays d'origine et leur pays d'accueil. L'étude combine une analyse de l'interface de présentation de soi sur Grindr et celle d'entretiens semi-guidés menés auprès d'un échantillon d'étudiants afin de déterminer comment ces individus déclarent qu'ils utilisent les affordances de l'application de rencontre pour projeter et entretenir une présentation de soi en ligne, interagir avec d'autres individus en déployant une gamme d'ajustements identitaires et sémiotiques et se confronter aux regards des autres. Les entextualisations (Jones, 2018) mobilisant des ressources textuelles et visuelles pour construire et entretenir son profil sont examinées afin de mettre au jour quelques-unes des stratégies d'exposition discursive des étudiants et les tensions identitaires que cela peut générer. D'une part, la visualité des applications de rencontres semble inciter les individus gays à se conformer à des normes valorisant la blanchité et la virilité. D'autre part, ces applications contribuent à la socialisation des étudiants étrangers mais les confronte à la dureté des rapports sociaux et à certains comportements de racisation.
\end{abstract}

\begin{abstract}
Situated in the field of digital migration studies, this research explores how international students use a dating application (Grindr) to present themselves and carve out an intermediary identity at the intersection of their countries of origin and the host country. The study combines an analysis of Grindr's interface where profiles are built and displayed and semi-guided interviews with 12 individuals in order to investigate how they create online versions of themselves, harness a range of identity and semiotic adjustments with a view to interacting with other individuals and confronting themselves to this online arena. Both the entextualisations (Jones, 2018) which mobilize textual and visual resources to build one's profile, and what users have to say about them are examined to uncover students' strategies of self-presentation and the identity tensions that can arise. On the one hand, the visuality of dating applications seems to incite gay individuals to conform to norms of whiteness and virility. On the other hand, these applications may facilitate students' socialisation but also confront them with the toughness of social relations and manifestations of racialization.
\end{abstract}




\section{Introduction}

Située dans le champ de recherche des études sur le numérique et la migration (Patterson \& Leurs, 2019), cette recherche porte une attention particulière aux discours médiés par le numérique (Paveau, 2017 ; Jones, 2018) et à certains enjeux identitaires liés à la migration des minorités (Diminescu, 2008 ; Shield, 2017). Une étude de cas a été conduite auprès d'étudiants étrangers ${ }^{1}$ gays usagers de Grindr, une application de rencontres pour les « hommes qui ont des relations sexuelles avec des hommes " (HSH), afin de comprendre comment ce groupe d'étudiants maniait les affordances $^{2}$ de cette app pour se présenter et se forger une identité intermédiaire entre leur pays d'origine et leur pays d'accueil. Comme le souligne Bergström (2016), «se saisir d'un dispositif technique, se mettre en scène dans un contexte de séduction, converser, donner de soi, exprimer son appréciation et faire des choses ensemble sont autant d'activités auxquelles concourent les usagers des sites de rencontres » (p. 37). L'hypothèse sous-tendant cette recherche est que les activités, parmi celles relevées par Bergström, mettent en jeu des aspects sémiotiques, sociaux et spatiaux dont l'étude peut élucider une partie du travail de construction identitaire à l'œuvre pour des individus gays engagés dans une migration. Comme le remarquent Bryant et Soria (2015) dans l'une des rares recherches consacrées aux étudiants étrangers $\mathrm{LGBT}^{+}{ }^{3}$, les séjours d'étude à l'étranger, en offrant l'occasion d'être dans un nouvel environnement à l'abri des contraintes culturelles du pays d'origine et du regard des proches, ménagent un certain degré de liberté pour explorer leur identité selon le niveau d'acceptation du pays d'accueil. Parmi toute une gamme de ressources sociales et technologiques, les applications de rencontres en ligne peuvent contribuer à cette exploration.

Se focaliser sur un échantillon d'étudiants étrangers gays fournit la possibilité de renouveler le regard sur des pratiques de rencontre devenues ordinaires (Bergström, 2016 ; Lardellier, 2012). Une telle étude permet également, à la suite de Leurs et Prabhakar (2018), de comprendre les relations qui se nouent entre l'expérience de migration - ici inscrite dans le cadre d'une mobilité académique plus ou moins longue - et des technologies numériques. Enfin, la particularité des médias de socialisation comme Grindr obligent ces individus, doublement en situation de minorité, à énoncer une identité et à confronter celle-ci aux regards des autres. Ainsi, cette étude postule que l'utilisation des applications de rencontre gay correspond à une pratique qui, parce qu'elle semble être à la fois porteuse d'émancipation et reproductrice de dominations, fournit un point d'observation privilégié pour examiner certains enjeux sociopolitiques liés la migration. L'examen de ces applications devrait ainsi permettre de mettre au jour certains régimes d'assignation en jeu selon des processus qui «rattachent, de l'extérieur, des individus à des catégories [qui] peuvent être ethnoraciales, de genre, mais aussi de religion, d'orientation sexuelle, voire liées au lieu de résidence, à l'âge ou à la situation de handicap » (Duvoux, 2017, p. 56).

Cette étude articule une analyse de l'interface de présentation de soi sur Grindr et l'analyse d'entretiens semi-guidés menées auprès d'un échantillon d'étudiants étrangers afin de déterminer comment ces individus déclarent qu'ils utilisent les affordances de l'application de rencontre pour projeter et entretenir une présentation de soi en ligne, interagir avec d'autres individus en déployant une gamme d'ajustements identitaires et sémiotiques et se confronter aux regards des autres.

\section{Positionnement et présentation des participants}

\subsection{Etudier les pratiques des migrants sur les applications de rencontres : positionnements}

Depuis les travaux de Diminescu (2008) sur les «migrants connectés », les recherches se sont multipliées pour explorer la place que tiennent les outils numériques dans l'expérience de migration. En effet, le constat a été fait que les smartphones, les médias sociaux et les applications sont utilisés par les migrants comme de nouveaux moyens d'accéder à de l'information et à des ressources diverses dédiées à la communication et à la gestion des émotions (Madianou et Miller, 2012), en particulier pour garder le lien avec les proches restés dans le pays d'origine et pour développer son réseau relationnel dans le pays d'accueil (Leurs et Prabhakar, 2018). Alors que la sexualité constitue un moyen très répandu de s'inscrire émotionnellement et socialement dans un pays d'accueil (Gray et Baynham, 2020), seules quelques études se sont intéressées aux aspects sémiotiques et identitaires que l'utilisation des applications de rencontre mettent en jeu pour des migrants gays (Patterson et Leurs, 2019; Shield, 2017) et aucune, à ma connaissance, ne concerne la catégorie des étudiants étrangers.

\footnotetext{
${ }^{1}$ Dans ce texte, le vocable étudiant étranger recouvre à la fois des étudiants engagés dans une mobilité courte (de quelques mois à une année) et des étudiants venus en France sans que le retour dans le pays d'origine soit forcément envisagé. L'appellation étudiant étranger sera employé plutôt qu'étudiant international qui appartient au vocabulaire technocratique de la mondialisation de l'enseignement supérieur et ne correspond pas à une catégorie opératoire en recherche.

${ }^{2}$ Cette notion, d'abord proposée par Gibson (1979), est employée dans l'acception qu'en propose Hutchby (2014) : « Les affordances sont à la fois fonctionnelles et relationnelles : fonctionnelles car elles permettent (et aussi contraignent) l'engagement dans une activité donnée en façonnant les possibilités associées à cette action. Elles sont également relationnelles en cela qu'elles peuvent différer pour un objet donné selon les contextes ou selon les espèces » [ma traduction].

3 Je reprends à Broqua (2018) le sigle LGBT+ pour désigner les individus se reconnaissant lesbiennes, gays, bisexuel.le.s, transsexuel.le.s . Comme le dit cet auteur, le + «permet de « couper court à la prolifération des lettres tout en restant fidèle au principe d'inclusion » (p. 154) et son ouverture signale une opposition de principe aux normes de genre majoritaires.
} 
L'intérêt de mener une étude sur les usages d'une application de rencontre auprès de la population des étudiants étrangers gays permet d'examiner comment des individus se positionnent à des degrés plus ou moins privilégiés sur une échelle de valeurs. En effet, dans ces espaces sociaux très particuliers que constituent les applications de rencontre, des facteurs tels que la nationalité, la religion, l'âge, l'orientation sexuelle et le statut migratoire peuvent se combiner pour influencer les perceptions vis-à-vis de ces individus et engendrer des marginalisations à plusieurs titres (Trawalé, 2016 ; Patterson \& Leurs, 2019). Ainsi, l'examen des pratiques médiées de présentation de soi conjugué à celui des jugements que posent les individus sur leurs pratiques de ces applications permettent de comprendre un certain nombre d'aspects identitaires et sémiotiques auxquels ils sont confrontés. Shield (2017) a ainsi produit une étude exploratoire sur l'usage de deux applications de rencontre (Grindr et PlanetRomeo) par des individus gays issus de l'immigration récente dans la région de Copenhague en examinant les textes de leur profil et en se focalisant sur les questions liées à l'exclusion lors d'entretiens afin de mettre au jour des dynamiques identitaires. Pour conduire des recherches sur usages d'une application de rencontre, certains chercheurs ont choisi de provoquer des données en collaboration étroite avec les usagers avec des techniques comme l'observation. Ainsi Batiste (2013) a suivi à la trace Jorge, un utilisateur toulousain de Grindr, sur plusieurs mois afin de comprendre comment les applications de rencontre permettent aux hommes gays de « reconfigurer des espaces sociaux hétéronormés » (p. 114, ma traduction) qu'ils s'approprient à travers des amitiés, des liaisons sexuelles et romantiques. Enfin, Patterson et Leurs (2019) ont conduit des entretiens avec de jeunes adultes gays (15-25 ans) qui ont choisi de migrer aux Pays-Bas de manière volontaire (situation d'expatriés) ou pour fuir leur pays parce qu'ils y étaient opprimés afin de déterminer les stratégies de socialisation déployées par le biais d'applications de rencontre, la formation identitaire et la gestion émotionnelle

L'approche choisie pour cette étude consiste en une analyse initiale de l'interface de Grindr pour identifier les affordances de cette application de rencontre géolocalisée et examiner les discours produits par et sur elle. En effet, comme le rappelle Develotte (2006, p. 88) :

\section{« l'exposition discursive [dans un espace médié] « conditionne » la production discursive : c'est en effet en fonction d'un espace d'exposition discursive spécifique que s'effectue la production d'un nouveau discours de la part d'un locuteur, sujet psychologique et acteur socialement situé ».}

Choisir de se focaliser sur une application de rencontre parmi d'autres applications possibles (par exemple Scruff, Tinder, PlanetRomeo) repose sur le principe qu'une interface donnée façonne les ressources sémiotiques des utilisateurs d'une manière spécifique et matérialise une idéologie culturelle (Mowlabocus, 2020). Ceci incite à ne pas considérer les outils ayant une même fonction (ici, trouver un partenaire proche physiquement) de manière générique mais au contraire en examinant soigneusement ce que chacun permet et contraint. Les travaux de Jones $(2011,2018)$ sur l'analyse du discours médié sur le langage et la sexualité ont montré qu'une affordance cruciale des applications numériques consiste à fournir des possibilités inédites de fabriquer des représentations entextualisées de soi. Jones (2011) définit l'entextualisation comme «le processus par lequel des actions sont transformées en moyens sémiotiques de médiation en vue d'actions à venir » (p. 288, ma traduction). Selon cette perspective, il s'agit de déterminer comment les technologies reconfigurent les situations sociales, les types d'action qui peuvent ainsi être entreprises ainsi que les identités qui peuvent être revendiquées ou construites. Cela signifie que les chercheurs intéressés par les pratiques médiées par une application donnée doivent se familiariser avec ses fonctionnalités afin de comprendre quelles actions sont possibles avec elle (Paveau, 2017). Toutefois, si les pratiques de drague sont en partie modifiées par ces applications de rencontre (Licoppe, 2016), leur examen doit maintenir un équilibre entre ce qui ressortit des affordances technologiques et ce qui appartient aux significations construites par des usagers, en l'occurrence de jeunes gays issus de minorités ethnoculturelles engagés dans un processus de migration plus ou moins long. Pour cette raison, l'examen de l'interface de présentation de soi sur Grindr entrepris pour cette étude est éclairé par les données recueillies lors d'entretiens conduits auprès d'un échantillon de 12 étudiants d'origine étrangère.

\subsection{Les participants}

Une enquête par entretien a été menée à l'automne 2019 avec des usagers de $\mathrm{Grindr}^{4}$ appartenant à la catégorie des étudiants étrangers, une catégorie d'étudiants dont les conditions sensibles de leur expérience à l'étranger ont été sensiblement modifiées par les outils numériques (Guichon, 2020). Selon King et Raghuram (2013), bien que les étudiants étrangers constituent une catégorie significative de la population migrante, cette migration reste relativement peu étudiée. Ces mêmes auteurs soulignent qu'une distinction doit être apportée entre les étudiants effectuant une mobilité de courte durée et ceux pour lesquels la mobilité académique constitue une potentielle porte d'entrée vers une migration définitive.

Les participants à cette étude, 12 étudiants d'origine étrangère, avaient entre 22 et 35 ans au moment de l'enquête avec un âge médian situé à 27,6 ans. L'échantillon présente une certaine diversité avec des étudiants venant principalement du continent américain (Colombie, Venezuela, Mexique, El Salvador, USA), du Moyen-Orient et du Maghreb (Syrie, Tunisie et Maroc, trois pays où l'homosexualité reste pénalisée) et d'Asie (Taïwan). Il est à noter que, malgré nos

\footnotetext{
${ }^{4}$ Je remercie Jean Carteron qui, dans le cadre de son mémoire de Master 2 à l'université Lumière Lyon 2, a contribué à recruter la plupart des participants, à conduire une partie des entretiens et à assurer leur transcription.
} 
efforts, nous n'avons pas réussi à recruter des étudiants venant de l'Afrique sub-saharienne, de Chine et du Brésil qui sont pourtant nombreux à venir étudier en France. Tous les participants s'identifiaient comme homosexuels mais présentaient différents degrés d'acceptation de leur propre sexualité évaluée sur une échelle de Likert allant de (1) "personne n'est au courant à l'exception de quelques proches» à (4) «tout le monde est au courant» selon un continuum proposé par Fox et Warber $(2015)^{5}$. Bien qu'imparfait, cet indicateur permet d'avancer qu'il faut posséder un certain niveau d'assurance pour participer à un entretien sur son utilisation d'une app de rencontres gay, ce qui peut expliquer la difficulté à recruter des étudiants venant d'aires géographiques et culturelles où l'homosexualité reste au mieux taboue ou au pire réprimée par loi.

Le profil ethnique - tel qu'il est déclaré sur Grindr et avec les dénominations que propose cette application - permet de constater une certaine diversité parmi les participants : asiatique (3), latino (5), Moyen-Orient (2), métis (1) et blanc (1). La durée de leur séjour en France varie entre quelques mois et huit ans, ce qui permet de distinguer, à la suite de King et Raghuram (2013), les étudiants en mobilité courte (un séjour d'étude allant de quelques mois à un ou deux semestres) et les étudiants migrants (au-delà d'une seule année avec une incertitude sur le projet de retour dans le pays d'origine), une variété qui permet de considérer différents types d'inscription dans le contexte d'accueil.

Les participants ont été recrutés par le biais de trois moyens : des affichettes placées dans un centre d'étude du français à Lyon (France), un profil créé sur Grindr explicitant la recherche et proposant d'y participer, et la mobilisation de proche en proche. La longueur des entretiens a varié de 42 minutes à $1 \mathrm{~h} 20$. Les participants ont signé un formulaire de consentement et ont choisi eux-mêmes un pseudonyme à même de préserver leur anonymat. Huit entretiens sur les douze ont été conduits en français, deux dans un mélange d'espagnol et de français et, enfin, deux complètement en anglais (avec les deux étudiants états-uniens). Les entretiens ont été retranscrits et ceux qui étaient en espagnol et en anglais ont été traduits. Comme le suggère Duff (2008) et parce que l'étude porte sur le contenu et non sur la forme, les marques de l'oral (pauses, hésitations, répétitions) ont été ôtées pour faciliter la lecture.

Tous ces participants ont été interviewés pour déterminer comment ils utilisaient les affordances de Grindr et comment ils se présentaient sur leur profil. Une partie de l'entretien était consacrée aux regards des autres et sollicitaient des participants des récits relatifs aux perceptions suscitées par leur couleur de peau chez les autres utilisateurs. Certains participants ont accepté de partager quelques échanges sur l'application et des captures d'écran afin d'ancrer les discours sur l'application par des échantillons de pratiques (cf. 3.1). L'analyse des entretiens a été structurée par les questions de recherche : les enjeux et les outils pour se présenter sur Grindr, les ajustements identitaires et sémiotiques pour les étudiants étrangers, et les questions liées au profilage ethnique inhérentes à ces applications.

\section{Analyses}

\subsection{Les affordances de Grindr comme dispositif d'exposition discursive}

L'objectif de cette première section consiste à envisager l'interface de présentation de soi de Grindr comme un « dispositif d'exposition discursive » (Develotte, 2006) qui contribue à façonner l'énonciation des utilisateurs grâce aux ressources d'entextualisation qui sont mises à leur disposition (cf. supra). Les énoncés produits en ligne sont considérés, à la suite de Paveau (2017, p. 40), comme «des composites technolangagiers co-intégrant pleinement la dimension technologique et la dimension langagière ». Pour se situer d'emblée dans la perspective de l'usager gay étranger, cette section va largement se focaliser sur les données obtenues auprès de Kyle, un états-unien d'origine philippine de 22 ans venu passer quelques mois à Lyon. Comme Maran, un autre états-unien de l'échantillon, Kyle constitue un bon exemple de la mobilité heureuse, celle d'un jeune issu d'une université prestigieuse d'Amérique du Nord qui peut se permettre de passer une année en Europe et tirer le maximum de cette expérience sur le plan intellectuel, social et hédoniste. Grindr constitue pour lui un outil de plus dans le répertoire du voyageur pour élargir efficacement son réseau social dans les pays qu'il visite. Le texte de son profil annonce «Je ne parle pas français. New to Lyon. Show me around? Cuddling is a plus ${ }^{6}$ 》, tressant ensemble plusieurs des aspects abordés dans cette étude, à savoir les éléments linguistiques, spatiaux et sexuels.

Grindr semble occuper une place primordiale dans la socialisation des gays comme le confirme Kyle qui reconnait être un usager régulier de cette application :

Grindr est le moyen principal pour moi pour faire des rencontres sexuelles (...) Je pense que son utilisation est tellement ancrée dans ce qu'être gay veut dire de nos jours que j'aurais du mal à trouver quelqu'un si je ne le trouvais pas par Grindr.

Grindr n'est donc pas seulement l'application de rencontres actuellement la plus populaire parmi les HSH, incontournable selon Kyle, mais une façon de s'approprier les codes de l'homosexualité à l'heure où les outils numériques contribuent à façonner non seulement les interactions avec les autres membres de la minorité gay mais à construire et à exposer une identité.

${ }^{5}$ Le continuum initial de Fox and Warber (2015) présente les items suivants : (1) "Mostly in the closet" (only a few people know);

(2) "Peeking out" (a wider audience than before knows); (3) "Partially out" (certain audiences know); and (4) "Out".

${ }^{6}$ Nouveau à Lyon. Faites-moi visiter. Câlins appréciés. 
L'examen des usages de Grindr permet d'identifier trois principales catégories d'affordances qui seront plus précisément discutées dans la suite du texte. En première instance, cette application permet de

- S'inscrire dans un territoire, partager, grâce à la géolocalisation, cette inscription avec d'autres utilisateurs appartenant majoritairement à la même minorité sexuelle, et évaluer sa place dans ce territoire partagé (indicateur de consultation du profil, messages reçus);

- Enoncer une identité avec les ressources d'entextualisation à disposition par le biais de l'interface de l'application avec d'une part des champs libres (photos, pseudos, textes) et des champs prédéfinis (origines, couleur de peau...) ;

- Interagir avec d'autres utilisateurs, soit pour les faire disparaître de la mosaïque des profils (blocage) soit pour initier les conditions pour une rencontre.

Pour présenter les affordances de cette application, la capture d'écran du smartphone de Kyle, prise lors de l'entretien dans un quartier aisé de Lyon, est présentée (cf. figure 1). Il faut d'emblée souligner que cet assemblage techno-humain serait différent si la capture d'écran avait été faite dans d'autres contextes (par exemple dans d'autres quartiers moins bourgeois de Lyon, en milieu rural, ou dans des pays où l'homosexualité reste pénalisée). Ceci souligne le caractère idiosyncrasique de ces configurations et des données qu'elles génèrent (Bibié-Émerit, 2016) qui varient non seulement en fonction du lieu où l'on se trouve au moment de leur utilisation et des filtres qu'on aura installés en amont sur son profil qui permettent de ne voir qu'une sélection parmi les usagers en ligne selon des critères définis (par exemple âge, rôle sexuel, caractéristiques ethniques). En outre, que l'utilisateur marche quelques temps et les profils changent, ce qui crée une tapisserie d'images numériques sans cesse renouvelée, la ville pouvant aussi s'explorer de cette façon.

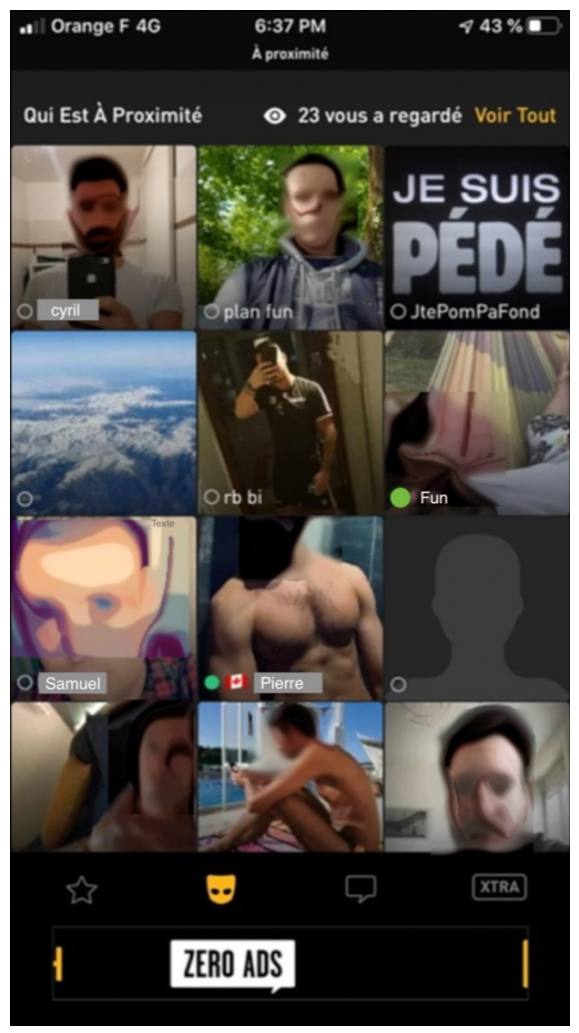

Figure 1. L'écran de Kyle le 7 janvier 2020 à $18 \mathrm{~h} 37$ dans le quartier de la Croix-Rousse à Lyon.

Comme on l'observe dans la figure 1 qui est organisé sous forme d'une mosaïque ${ }^{7}$, les profils des utilisateurs sont disposés par rangées de trois selon leur proximité physique avec le propriétaire du smartphone, le plus proche au moment de la capture d'écran étant en haut à gauche. L'accès à l'autre se fait par le biais d'une image-lien, ce qui place la mosaïque de portraits au cœur des interactions entre les usagers et la mise en scène de soi comme un enjeu critique pour attirer l'attention. Comme le dit Kyle :

\footnotetext{
${ }^{7}$ La capture a été retouchée afin d'anonymiser les profils tout en conservant les caractéristiques sémiotiques du dispositif textuel (pseudonymes modifiés) et visuel (visages floutés).
} 


\begin{abstract}
L'appli est indissociablement ancrée dans la visualité et je trouve cela problématique (...). $C$ 'est comme pour Instagram : tu te mets des filtres, tu prends des poses, tu prends des photos de toi d'une certaine manière et il y a une bonne façon de faire tout cela de telle sorte que tu obtiennes la meilleure version de comment quelqu'un te perçoit à travers tes photos. Beaucoup de profils annoncent "pas de pics, pas de dial".
\end{abstract}

Même s'il est restreint de facto à la communauté d'HSH, Grindr semble perçu comme un média social qui place la présentation visuelle de soi comme condition sine qua non à la participation pleine et entière, une condition annoncée par le message « pas de pics, pas de dial » (pas de photos, pas d'échanges) affiché dans un grand nombre de profils. L'image est donc au centre de la plupart des actions. Une fonctionnalité permet de savoir combien de personnes ont consulté un profil (ici on voit « 23 vous a regardé »), ce qui constitue un moyen de mesurer son attractivité et de situer dans la « hiérarchie du désir» (cf. infra). En cliquant sur l'image d'un profil, on peut voir la distance physique exprimée en mètres, lire la fiche de la personne avec les informations le concernant (pseudonyme, un court texte descriptif, taille, poids, origine ethnique, type de corps (par exemple musclé), les «tribus » dans lesquels l'individu se reconnaît (par exemple celle des «bears » qui désignent habituellement des hommes poilus et costauds), s'il est ou non engagé dans une relation amoureuse, ce qu'il recherche et, enfin, un lien éventuel vers trois réseaux sociaux (Twitter, Facebook et Instagram). Toutes les informations ne sont pas forcément renseignées (il semble courant que l'âge ou le statut amoureux restent vides) et aucune n'est vérifiée. Les menus déroulants contraignent certains choix pour se décrire obligeant par exemple à sélectionner une catégorisation ethnique parmi un choix limité, ce qui enferme les individus dans une assignation identitaire selon des normes euro-américaines (cf. infra). Le pseudo, le texte libre de description et la photo sont laissés à l'appréciation des utilisateurs et ménagent une place à la création d'une identité intermédiaire.

Parmi les douze profils de la figure 1 examinés à titre d'exemple, se dessine un nuancier sur le thème de la présentation de soi sur une application de rencontre gay : les utilisateurs fixent de potentiels interlocuteurs, les sourcils plus ou moins froncés, ou bien regardent ailleurs ; ils affichent, à des degrés divers, visages et corps ou les soustraient aux regards ; leur pose est plus ou moins figée, naturelle et plus ou moins sexualisée. Une grammaire de la présentation de soi semble s'être construite avec des codes visuels contribuant à survaloriser la masculinité (musculature apparente, visage fermé, expression détachée), comme le confirme Kyle :

Esthétiquement, il y a une sorte de modèle. C'est soit le plan torse sans visage, et c'est encore mieux s'il est torse nu [rire], ou alors le visage sérieux. C'est rare de voir un sourire. Je ne sais pas si tu remarques mais il y a toujours ce regard déconnecté sur leur visage. (...) Et puis, il y a les selfies; ce ne sont pas des selfies qui exsudent le bonheur selon moi. Ce sont des selfies qui projettent une masculinité dure.

Comme on l'observe dans la figure 1, les photos choisies peuvent révéler une partie du contexte des utilisateurs (chez soi, dans la nature, à la piscine, devant un endroit connu) ou au contraire saturer l'image du seul contexte (dans l'avion au-dessus des Alpes). Le contexte permet non seulement de dévoiler des informations (les voyages, les pratiques sportives) mais de replacer les corps parfois dénudés dans le cadre de pratiques sociales dessinant ainsi un continuum dans lequel la sexualisation est plus ou moins centrale ou périphérique.

Enfin, si l'on regarde les pseudos qui constituent un élément définitoire de la drague en ligne (Lardellier, 2012), ils permettent de compléter l'assemblage technographique (Paveau, 2017) de présentation de soi en se combinant avec la photo principale du profil. Plusieurs possibilités dans le maniement de cette affordance textuelle sont repérables sur la capture d'écran : afficher un prénom, sa nationalité (par le biais d'un drapeau), une orientation sexuelle (bi), expliciter ses intentions (plan fun) ou au contraire suspendre ce surtitrage de soi par un espace laissé vide.

Pour résumer, cette association (texte + photo) permet de projeter une identité plus ou moins authentique (prénom et nationalité révélés, visage apparent), une apparence plus ou moins travaillée (filtre, mise en scène), un dévoilement social plus ou moins important (avec ou sans contexte), des intentions plus ou moins focalisées sur une gratification sexuelle immédiate ou au contraire laissant entrevoir d'autres types d'interaction (amitié, amour).

Enfin, la consultation d'un profil peut conduire à plusieurs actions : on peut initier un échange minimal - un tap signalant un intérêt pour un individu, entrer en interaction avec lui par le biais du chat écrit, l'inscrire comme favori pour pouvoir retrouver le profil et communiquer facilement avec celui-ci ultérieurement. Mais on peut également ignorer une personne qui souhaite entrer en contact ou bien encore le bloquer pour empêcher tout échange ultérieur. Mowlabocus (2020) insiste sur le fait que filtrer des catégories d'individus parmi les profils visibles de la mosaïque (par exemple des personnes de couleur ou dépassant un certain âge) ou bloquer des individus correspondent à des affordances de ségrégation furtive qui permettent de faire littéralement disparaître certains individus de manière plus ou moins consciente de son espace.

Si les affordances de Grindr (inscription et exploration territoriales, énonciation d'une identité, interaction avec d'autres individus) ont été examinées séparément, c'est bien leur combinaison qui constitue une ressource spécifique qui peut être choisie pour développer une entextualisation de soi et accompagner l'exploration du contexte hôte. Comme le dit Rahim (Maroc, 25 ans) : « [avant de partir du Maroc], je savais pas qu'il y avait cette application. C'est un ami gay de République Tchèque, quand il a été au courant que je venais en France, il m'a dit 'dès que tu arrives, tu installes Grindr' ». Pour Rahim comme pour les autres participants à cette étude, utiliser Grindr en arrivant en France constitue un moyen pour trouver une place à la fois dans une communauté sociale et dans une communauté de discours et suppose d'apprendre à manier les ressources sémiotiques et de s'approprier un certain nombre de codes. 


\subsection{Prendre sa place dans la compétition du désir : " comme un casting »}

Un des enjeux pour quiconque se présente sur une application de rencontre consiste à capter l'attention dans un flot visuel et textuel d'autant plus intense que l'utilisateur se trouve dans un grand centre urbain. Dans leur analyse interactionnelle des échanges sur Grindr, Licoppe, Rivière et Morel (2016) montrent que les échanges suivent un script standardisé dédié à aboutir rapidement à une rencontre (un plan) et privilégiant généralement la dimension sexuelle sur d'autres dimensions plus personnelles. Luis (35 ans, Venezuela), un des participants à cette étude, décrit cet enjeu et les échanges stéréotypés sur Grindr comme une sorte de casting ("c'est bref comme un casting, super bref »). Licoppe et al. indiquent en effet que les échanges sur Grindr sont généralement courts et les séquences d'ouverture s'éloignent autant que possible des conversations ordinaires pour se résumer généralement à une série stéréotypée de questions destinées à vérifier la compatibilité sexuelle et la disponibilité. Rahim ( 25 ans, Marocain) décrit ainsi la tournure habituelle des échanges sur l'application : "Salut la forme ? Ensuite bah qu'est-ce que tu racontes ? Tu fais quoi de beau sur Lyon ? Tu cherches quoi sur l'appli ? Et puis... ». Les échanges fonctionnent comme « des checklists mutuelles dans lesquelles la vérification de la compatibilité des critères remplace l'évocation et la discussion d'événements personnels » (Licoppe et al., 2016, p. 2549, ma traduction). Maran, un des participants états-uniens de 28 ans, décrit ainsi cette interaction : «J'ai ça, ça, ça et ça. Ah, il semble que tu aies un, deux et trois mais tu n'as pas le numéro 4. On annule. Tu dégages. Va te trouver quelqu'un d'autre ». Comme on le constate grâce à l'effet de loupe que produit Grindr, les utilisateurs connaissent les règles de la drague qui sont parfois impitoyables pour qui ne possèdent pas les attributs les plus demandés et disposent donc d'un «capital érotique » (Green, 2013) moindre. Les utilisateurs apprennent donc à se mettre en valeur selon les codes auxquels ils se conforment comme Andrès (33 ans, Mexicain) le confirme en expliquant la construction de son profil et le choix des photos : «Oui, forcément, c'est pour attirer. C'est comme quand on est dans un supermarché et qu'on lit l'étiquette. Donc c'est comme ça ». Hobbs, Owen et Gerber (2017) ont relevé cette tendance, souvent acceptée, qui conduit l'utilisateur d'une application de rencontre à se traiter soi-même comme une marchandise dont il faut travailler l'image pour la rendre attirante afin de pouvoir prendre sa place dans la compétition du désir.

Cascalheira et Smith (2019) ont mis en place une étude expérimentale en psychologie sociale pour déterminer selon quels biais ethniques (blancs, noirs, asiatiques, latinos) et hétéronormés (se conformant plus ou moins à l'apparence supposée masculine d'un homme hétérosexuel) les HSH sélectionnent de potentiels partenaires sur des applications de rencontre. Comme le rappellent ces auteurs, l'une des particularités des applications de drague est de pouvoir filtrer les profils auxquels les utilisateurs sont potentiellement confrontés selon des critères ethniques ou de rôle sexuel (actif, passif, versatile). Les utilisateurs font défiler les profils et, sur la base d'un jugement construit sur des indices principalement visuels, ils choisissent d'entrer ou non en interaction avec tel ou tel partenaire. Dans leur étude, 395 participants étaient sondés par le biais d'un questionnaire en ligne qui simulait l'usage d'une application de rencontre et les invitait à opérer une sélection parmi des profils qui avaient été sélectionnés en amont afin de représenter une variété de profils ethniques et de degrés de masculinité (musclé vs mince, poilu vs imberbe). Il ressort que, quelle que soit la provenance ethnique des répondants, les catégories de profils les plus en demande sont celles d'hommes d'apparence masculine avec des traits caucasiens. Cascalheira et Smith concluent leur étude en avançant qu'une hiérarchie du désir basée sur des normes hétérosexuelles s'est mise en place sur ces applications de rencontre destinées aux HSH.

La relégation des hommes non blancs et d'apparence moins masculine au second rang de la hiérarchie du désir est-elle ressentie par les participants à cette étude ? La centration sur la visualité dans les échanges sur Grindr conduit certains participants à questionner les canons valorisant le corps musclé plutôt que la personnalité comme le remarque Huang (30 ans, Taiwan) :

«De plus en plus de gays font de la musculation. C'est la tendance. Tout le monde. En Occident, en Asie, c'est la même situation. Je n'aime pas ça. Parce que je pense qu'il y a beaucoup de choses qui constituent un gay. Chez un homme tu as beaucoup d'aspects comme le talent, les loisirs, les pensées.

Je déteste les gays qui se concentrent sur leur corps ».

Toutefois, il semble que la position de Huang soit minoritaire et que la primauté de l'image, exacerbée par les applications de rencontre, soit acceptée comme un mal nécessaire comme le relève Rahim (25 ans, Marocain) : « C'est drôle parce que tu vois, les photos, c'est super important sur Grindr. C'est super superficiel et en même temps, les gens, ils peuvent se fier qu'à ça ». Cela souligne que, dans leur majorité, les gays semblent accepter un discours dominant (ElTayeb, 2012; Vörös, 2018), façonné par les normes hétérosexuelles, qui conceptualise une identité gay fondée sur les attributs de la virilité et de la blanchité et qui impose de se confronter, voire de se conformer quand cela est possible, à ces règles du jeu. L'image projetée sur Grindr acquiert donc une valeur étalonnée selon une hiérarchie qui assigne à chaque utilisateur une place particulière dans cette économie du désir selon une logique néolibérale. En conséquence, les corps qui ne correspondent pas aux modèles idéaux (trop gros, trop vieux, trop minces, trop imberbes, trop féminins, trop colorés, trop éloignés d'une norme euro-américaine) placent une grande majorité de leur propriétaire en situation d'exclusion de la communauté et les relèguent à une situation « de gays qui n'ont pas ce qu'il faut pour être vraiment gay » pour reprendre la formule d'El-Tayeb (2012). Si le développement d'une identité gay selon les normes néolibérales du Nord global semble désirable pour la plupart de ces individus en mobilité, atteindre cet objectif dépend de leur capacité et de leur volonté à adopter certains codes de présentation de soi qui leur permettent de prendre leur place dans la compétition du désir telle qu'elle s'organise sur des applications comme Grindr. 


\title{
3.3 Ajustements identitaires et sémiotiques : « tout un travail sur soi »
}

Chaque usager des sites de rencontre en ligne doit apprendre à procéder à une «présentation textuelle et visuelle de soi » (Bergström, 2016, p. 19) en se profilant et en mettant en récit son identité sociale. Retenir efficacement l'attention et fournir des accroches pour amorcer une interaction par le biais du profil constituent deux enjeux cruciaux pour les utilisateurs. Le fait de se distinguer par des traits singuliers au milieu d'une communauté d'utilisateurs majoritairement blancs peut complexifier la présentation de soi pour les étudiants étrangers qui ont en outre intériorisé la hiérarchie du désir mise au jour plus tôt.

Une réflexion pour mettre sa présentation en conformité avec les codes de l'espace de drague en ligne se construit progressivement et se concrétise par la production de différentes versions de soi :

\begin{abstract}
"Mon profil est beaucoup plus garni quand même qu'au début. J'ai eu le temps de me chercher et aussi de savoir ce qui me plaît. Franchement les photos, c'est tout un travail sur soi. Parce qu'en arrivant ici, je me prenais absolument pas en photo. Je n'aimais pas. Je ne m'assumais pas physiquement. Au fur et à mesure, j'ai appris à faire des photos de mieux en mieux et voilà. (...) J'ai pris plusieurs photos qui me plaisent bien, de moi, de mon visage. J'en ai une torse nu parce que forcément les gens ils veulent voir. J'essaye de faire en sorte que les photos montrent que je suis approchable, sympa, une personne avec qui c'est très facile de discuter. (...) Voilà j'ai pas absolument tout rempli mais j'ai rempli le nécessaire pour que ça fasse pas un profil vide. Parce que les gens, quand c'est tout vide, ils se demandent pourquoi ». (Rahim, 25 ans, Marocain).
\end{abstract}

Les stratégies pour se présenter sur Grindr requièrent de faire «tout un travail sur soi », comme le dit Rahim, un apprentissage qui est autant de nature personnelle (accepter son corps et son identité sexuelle) que sémiotique (rendre un profil attractif en prenant des photos valorisantes, dévoiler sa personnalité et ses atouts physiques avec les outils visuels et textuels à disposition). Jones (2018) insiste sur le fait que « la façon de développer une identité sexuelle à soi est intimement liée aux possibilités offertes par les outils culturels pour déployer une identité textuelle propre » (p. 10, ma traduction). Dans la plupart des cas, ce travail de présentation de soi (apprendre à plaire) s'accompagne d'une connaissance de soi-même et d'un regain d'estime de soi (apprendre à se plaire) comme on le perçoit bien dans le discours de Rahim qui a appris à aimer son physique depuis son arrivée en France en le rendant visible sur Grindr.

La discontinuité relative à l'utilisation des applications de rencontre avant et après l'arrivée dans le pays hôte est souvent commensurable à la situation des homosexuels dans les pays d'origine des participants. Pour les étudiants qui viennent des pays du Nord global, les changements concernant la présentation de soi avant et après l'arrivée en France sont minimes. Ainsi, Kyle (Etats-Uniens, 22 ans) a conservé sur son profil les mêmes photos de lui le montrant devant différents endroits aux Etats-Unis comme le Musée Guggenheim à New York ou en France (château de Versailles) : ces décors fonctionnent comme autant d'indices contextuels qu'il possède un capital social et culturel dont la valeur est immédiatement reconnaissable. En revanche, pour les étudiants qui viennent de certains pays du Sud global où l'homosexualité reste stigmatisée ou pénalisée, il s'agit de passer d'une situation clandestine ou quasi clandestine à un dévoilement progressif de son identité, d'abord à soi-même puis aux autres.

L'espace de drague en ligne est non seulement « un espace où se teste et s'exerce la capacité de séduire » mais où l'on peut éprouver un sentiment d'appartenance à une sociabilité, à un 'milieu » gai' » (Vörös, 2018, p. 200). La pratique de cet espace suppose de dire quelque chose de soi et de tisser une narration dans laquelle l'identité pourra être ajustée au nouveau contexte du pays hôte. C'est le cas d'Alonso (27 ans, Mexicain) qui a décidé de franciser son nom : la superposition de son visage et du prénom français choisi (Alain) permet selon lui de dérailler le script stéréotypé évoqué plus haut pour amorcer un échange plus significatif ("Alors là, on peut parler plus »). On peut gager que lorsqu’Alonso décide de se renommer Alain, ce n'est pas d'une simple traduction qu'il s'agit mais de l'adoption d'une identité à michemin entre le pays d'origine et le pays d'accueil. La question de quelle identité (ethnique, culturelle et linguistique) revendiquer se pose fréquemment quand il s'agit de remplir les champs de l'interface de Grindr; ainsi Estéban (27 ans, Colombien) se choisit une couleur de peau (blanche) qu'il admet légèrement différente de la réalité. En outre, il gomme ses origines en affichant le drapeau espagnol plutôt que colombien non seulement pour indiquer qu'il est hispanophone mais pour rehausser symboliquement son image, l'Espagne étant selon lui plus désirable que son pays d'origine où les gays continuent d'être la «honte de [leur] pays ». Des tentatives d'auto-blanchiment ou d'européanisation de l'identité sont donc détectables dans certains profils témoignant peut-être d'une volonté de se réinventer, ou bien de se conformer aux perceptions de la population. La hiérarchie du désir se conjugue avec une hiérarchie sociale avec laquelle les étudiants apprennent à composer.

Certains étudiants gays présentent une sorte d'identité éclatée comme Andrès (33 ans, Mexicain) qui distribue les indices le concernant (sa barbe, sa peau mate, ses jambes imberbes) dans différentes photos dans lesquelles son visage n'apparaît que de biais. Conscient des regards qui peuvent être posés sur lui («tout le monde n'aime pas la peau mate »), Andrès révèle suffisamment d'indices pour maintenir, malgré l'anonymat, un certain niveau d'authenticité sans dévoiler l'ensemble de son identité On constate en outre que les photos utilisées pour garnir le profil de Grindr ont parfois initialement été postées sur des réseaux sociaux (Facebook, Instagram, Twitter) où leur potentiel a pu être testé et validé. Il s'agit alors de jouer avec habileté avec les codes de la drague en ligne et d'exhiber selon, sa personnalité et l'image que l'on souhaite projeter, sa virilité, sa beauté, sa sportivité, son caractère tout en prenant soin de montrer « patte blanche» (« je suis approchable» (Rahim, Marocain)), «Je choisis des photos normales » (Assad, Syrien)) comme s'il était autant question d'attirer le regard que de rassurer. 
Les outils numériques permettent donc de polir son image à loisir et de dire ce que l'on veut tant que cela demeure crédible d'autant que la véracité des caractéristiques projetées (couleur de peau, âge, forme physique) est soumise à la contrainte d'une rencontre physique (Blackwell, Birnholtz et Abbott, 2014). L'authenticité dépend de l'adéquation entre l'image projetée sur l'application et l'image réelle avec juste ce qu'il faut de retouches, d'ajustements et de dissimulation pour attirer l'attention sans risquer de se singulariser trop ou d'être perçu comme un imposteur. Ainsi, les étudiants étrangers gays s'essaient à être eux-mêmes, pour reprendre la formulation d'Assad; le polissage sémiotique de l'identité projetée implique pour ces individus de se mettre en chantier par petites touches textuelles et visuelles afin de produire des versions sans cesse retravaillées d'eux-mêmes. Les entextualisations ainsi produites sont sans doute précieuses pour expérimenter une nouvelle liberté à bonne distance des regards et des normes familiales, culturelles et sociales de leur pays d'origine (Collins, 2012) même si des tensions, parfois exacerbées par le fait de devoir énoncer et socialiser une identité selon les contraintes d'un espace normé, se font jour.

\subsection{Tensions identitaires : « de mettre tes origines en avant, ça me dérange »}

Cette dernière section va aborder plus précisément la question de la place des étrangers dans un espace médiatique qui compte, dans une ville comme Lyon, un nombre moindre de profils de personnes minorisées. Dans son approche phénoménologique de la blanchité, Ahmed (2007) souligne le paradoxe suivant : les corps non blancs sont rendus invisibles lorsqu'ils apparaissent dans des espaces à dominante blanche en même temps qu'ils sont hyper-visibles car ils se démarquent des autres corps environnants. De facto, quand ils utilisent Grindr dans une ville comme Lyon, les étudiants étrangers se trouvent dans une situation de minorité et on peut faire l'hypothèse que la singularité des profils qu'ils présentent peut avoir un impact sur leur expérience de socialisation en ligne. Dans la section précédente, nous avons vu que les étudiants étrangers gays se réinventaient en partie une identité grâce aux affordances de Grindr. Mais le travail identitaire est forcément une coproduction entre celui qui se prête aux regards et ceux qui regardent et font défiler les profils selon des critères qui ne sont pas toujours conscients (Blackwell et al., 2015).

Interrogé sur le profil ethnique qu'il doit renseigner sur Grindr, Salah, un étudiant tunisien de 23 ans, dont le pseudo sur Grindr est « intello sérieux », déclare :

\section{«Pour l'ethnicité, j'ai changé à Moyen-Orient, bien que ça ne me définisse pas. Avant, je mettais " autre " mais j'ai vu que toutes les personnes du sud de la Méditerranée, ne mettent pas méditerranéen (...). Après y a des personnes qui fantasment sur les rebeus. (...) Comme c'est écrit " homme du Moyen- Orient », des fois certaines personnes attendent la personne en survêt un petit peu wesh wesh. (...) Ils fantasment sur ce type de personnes, pour certaines choses. Le fait de mettre tes origines en avant, ça me dérange ».}

On voit dans le discours de Salah que Grindr conduit à une racisation des utilisateurs non blancs en les réduisant de facto à leurs traits et leur couleur de peau (Guillaumin, 1992 ; Eid, 2018). L'application oblige à choisir son groupe ethnique (cf. 3.1), à mettre en avant ses origines, même si on ne se reconnaît pas dans celles qui sont proposées car elles défient parfois les réalités culturelles et géographiques. Ayant d'abord préféré l'indéfinition (autre) pour décrire son appartenance ethnique, mais craignant que cette indéfinition se retourne contre lui, Salah a fini par se conformer aux catégorisations imposées (Moyen-Orient ou méditerranéen) en regardant comment d'autres, semblables à lui, ont accepté de se couler dans une catégorie impropre dans laquelle il ne se reconnaît toutefois pas (ça ne me définit pas). En juxtaposant la photo et la description ethnique, Grindr entretient les stéréotypes chez ceux « qui attendent la personne en survêt un petit peu wesh wesh ": le Nord-Africain devient forcément le rebeu, objet de fantasme, affublé d'une tenue de sport générique et doté d'un langage de banlieue stéréotypé. L'étiquette ethnique imposée non seulement ne définit pas Salah mais elle l'assigne à une identité sociale de "mec de la cité » qui ne correspond pas à cet individu qui se décrit lui-même comme un « intello sérieux ». Il faut sans doute rappeler ici que Grindr est un dispositif sociotechnique qui a été façonné par des blancs - conçu par des Américains et lancé sur le marché mondial en 2009 - pour des blancs. Dans un tel dispositif, le non-blanc rompt la familiarité, en tout cas dans les pays à majorité blanche. Car comme le rappelle Ahmed (2007), le colonialisme blanchit le monde et nous prédispose à un certain type de corps.

A plusieurs reprises dans l'entretien, Salah se défend d'être l'objet de racisme sur Grindr. Pourtant, certains des échanges sur Grindr que Salah rapporte s'apparentent à une naturalisation de la différence (« Ah tiens, t'as pas des cheveux d'arabe », remarque l'un de ses interlocuteurs). Plus tard dans l'entretien, Salah raconte comment il a entretenu un échange nourri sur Grindr avec un voisin dans sa résidence universitaire pendant trois jours jusqu'à ce que Salah lui dise qu'il n'était pas français mais tunisien avec pour effet immédiat que « ça a refroidi la conversation ». De l'identité confortable car connue du «rebeu», le glissement soudain de Salah vers une identité trouble peut installer de l'inconfort puis, éventuellement, le silence. On constate enfin que l'interaction peut se rompre sur-le-champ car les fonctionnalités techniques (blocage) et les codes des interactions médiés par les technologies le permettent (Batiste, 2013). Grâce à son potentiel de géolocalisation, Grindr crée ainsi des proximités spatiales inattendues auxquelles il est néanmoins facile de se dérober en bloquant le profil qui dérange et qui disparaît aussitôt du champ des visibles.

Le témoignage de Salah souligne la dimension politique d'être ainsi exposé et étiqueté et on voit que le regard peut glisser sur les corps qui, par leur couleur, ne correspondent pas aux canons attendus ou au contraire s'y arrêter parce qu'ils ne semblent pas à leur place. L'étude des applications de rencontre conduit ainsi à examiner les régimes de visibilité dans les espaces en ligne (Barbosa Gadelha, 2016) et souligne que la centration sur la visualité accentue les 
risques d'essentialisation courants sur les applications de rencontre gay (tous les latinos sont hot) ou de racisme (pas d'asiats svp. Je n'ai rien contre eux mais ce n'est juste pas mon kif). Ainsi Rahim (Marocain, 25 ans) raconte les messages qu'il reçoit : «Clairement quand t'es rebeu, on vient te voir, on te dit '[...] Est-ce que tu es dominant, super viril ?' Enfin tout le délire du rebeu de cité ». Il semblerait d'une part que la dépersonnalisation des applications de drague en ligne désinhibe certains discours qui assignent aux membres des groupes minoritaires « une 'essence' propre, productrice de conduites et de qualités spécifiques inscrites dans la chair et le sang » (Guillaumin, 1992, p. 13-14). Ces discours banalisés portant sur les attributs physiques et les comportements sexuels stéréotypés, confrontent donc les individus de couleur à une racisation répétée avec son lot de fétichisations et de rejets assumés (stigmatisations) ou subreptices (blocage et filtrage de certains profils).

La mobilité étudiante et les outils et applications qui accompagnent celle-ci peuvent certes constituer une opportunité pour les étudiants étrangers gays pour se réinventer loin des normes culturelles de leur pays d'origine et s'adonner aux plaisirs de la séduction. Toutefois, comme on vient de le voir dans cette dernière section, il convient de se garder de tisser un récit d'une libération sans condition pour ces individus qui viennent séjourner en France et parfois s'y installer. Il faut également se garder de considérer les applications de rencontre comme des espaces dans lesquels toutes les expressions identitaires sont reçues avec bienveillance. Si, comme l'atteste le corpus d'étude, ces étudiants gays fabriquent de nouvelles versions d'eux-mêmes par leur biais, le processus de réinvention, voire d'émancipation, reste soumis aux regards des autres qui, avec quelques tapes sur un écran de smartphone, peuvent reconnaître et valider cette image exposée ou bien au contraire choisir de la rendre invisible.

\section{Conclusion}

Cet article a examiné le travail de présentation de soi déployé par un échantillon d'étudiants étrangers gays sur Grindr, une plateforme de rencontre aujourd'hui devenue "une institution de la culture gay» (Mowlabocus, 2020); l'entextualisation au moyen de ressources textuelles et visuelles mobilisées pour construire et entretenir son profil renseigne sur quelques-unes des stratégies des étudiants étrangers pour prendre leur place dans cet espace d'exposition discursive et sur les enjeux identitaires auxquels ils sont confrontés. Une variation relative aux déplacements identitaires identifiables sur leur profil Grindr se fait jour : les ajustements sémiotiques sont moindres pour ceux des étudiants qui sont de passage, surtout s'ils bénéficient d'un capital symbolique fort comme les deux états-uniens de l'échantillon, tandis qu'ils sont plus conséquents pour ceux des étudiants qui sont engagés dans un cycle d'étude long voire dans un projet de migration. A certains égards, l'expérience de migration semble facilitée par les applications de rencontre en ménageant des possibilités nombreuses d'interaction avec des individus du pays hôte appartenant à la même minorité sexuelle et en fournissant un observatoire sur les codes de cette communauté de discours. Ces applications offrent en outre des ressources à chaque utilisateur pour créer une version de soi-même adaptée au pays d'accueil et progressivement façonnée par les échanges avec les autres utilisateurs. Bien entendu, ces applications ne constituent qu'une ressource de socialisation transnationale parmi d'autres parmi d'autres ressources en ligne ou hors ligne. Les groupes mis en place et animés par des membres de diasporas sur les réseaux sociaux jouent un rôle potentiellement plus important en fournissant un accès à toute une communauté susceptible d'aider les nouveaux arrivants (Diminescu et Loveluck, 2014). Toutefois, parce que l'homosexualité continue d'être perçue comme une déviation par rapport aux normes culturelles, religieuses ou politiques dans beaucoup des pays qui envoient des étudiants vers des universités européennes, les applications de rencontre gay fonctionnent comme un outil de mobilité augmentée en constituant un accélérateur de socialisation au sein d'une enclave gay.

Se joue dans les échanges sur ces applications une dialectique entre visibilité (l'image que l'individu affiche pour attirer l'attention de potentiels partenaires) et invisibilité (généralement liée au degré d'acceptation de son orientation sexuelle par des individus provenant de pays où l'homosexualité reste encore criminalisée ou taboue) et toutes les stratégies intermédiaires de dévoilement et de cache-cache qui font de l'utilisation de ces applications une pratique à fort potentiel fantasmatique. Se profile également une pression, intimement liée à la visualité des applications de rencontres et à leur logique de marchandisation de soi, qui incite les individus gays étrangers à se conformer à des stéréotypes physiques valorisant la blanchité et la virilité. Le processus de polissage sémiotique de l'identité projetée par ces individus dans ces espaces en ligne ménage peu de place à la subversion de ces codes : les adopter et les maitriser conditionnent de conserver sa place dans la mosaïque des profils visibles sur Grindr et de ne pas entamer son capital érotique. Notons d'ailleurs que l'échantillon limité de participants à cette étude n'a pas permis d'inclure de présentations de soi transgressant ces codes alors même qu'il serait nécessaire d'examiner des subjectivités dissidentes des migrants queer à l'ère néolibérale comme nous y invitent Provencher et Peterson (2020). Enfin, l'utilisation de ces applications confronte les étudiants étrangers d'une part à la dureté des rapports de séduction soumis à une hiérarchie du désir défavorable aux étudiants non-blancs et d'autre part à certains comportements de racisation facilités par l'anonymat que procure les applications. Le profilage ethnique que Grindr met en place en demandant à chaque utilisateur de s'auto-assigner à un groupe ethnique et en permettant de filtrer les utilisateurs visibles selon ces mêmes catégories ethniques complexifie la tâche pour ces utilisateurs pour trouver leur place au sein d'une majorité blanche. ${ }^{8}$

\footnotetext{
${ }^{8}$ A la suite de l'assassinat de George Floyd par étouffement au printemps 2020, il est d'ailleurs intéressant de noter que Grindr a annoncé son intention de revoir sa politique d'assignation ethnique même si cette annonce n'a pas été suivie d'effets à l'heure où cet article est publié
} 
Pour finir, au-delà des pratiques de rencontre qui offrent un point de vue particulier sur l'expérience de migration, cette étude enjoint à examiner les enjeux liés à l'accueil des étudiants migrants selon une approche intersectionnelle sensible aux dynamiques de hiérarchisation (Trawalé, 2016) - raciale, genrée, sexuelle, socioéconomique - construites et reproduites vis-à-vis de certaines populations minoritaires. Selon cette approche intersectionnelle, l'examen des pratiques numériques et des perceptions des acteurs sur certaines de leurs pratiques pourrait grandement contribuer à mieux comprendre certaines inégalités qui se jouent dans l'expérience de migration, que celle-ci concerne les étudiants étrangers ou d'autres catégories de migrants.

\section{Références}

Ahmed Sara. (2007). A phenomenology of whiteness. Feminist Theory, 8, 149-168.

Barbosa Gadelha Kaciano. (2016). The Persistence of Desire: Gay Men Online and Queer Visuality. Arte y Politicas de Identidad, vol. 15, n 15, p. 59-72. [http://search.proquest.com/docview/1920222431/]

Batiste Dominique. (2013). 'O Feet Away': The Queer Cartography of French Gay Men's Geo-social Media Use. Anthropological Journal of European Cultures, vol. 22, n 2, p. 111-132.

Bergström Marie. (2016). (Se) correspondre en ligne: L'homogamie à l'épreuve des sites de rencontres. Sociétés contemporaines, vol. 104, n , p. 13-40. doi:10.3917/soco.104.0013.

Bibié-Émerit Laëtitia. (2016). La notion de lieu de corpus : un nouvel outil pour l'étude des terrains numériques en linguistique, Corela, vol. 14, $\mathrm{n}^{\circ} 1$ [http://corela.revues.org/4594]

Blackwell Courtney, Birnholtz Jeremy, Abbott Charles. (2015). Seeing and being seen: Co-situation and impression formation using Grindr, a location-aware gay dating app, New Media and Society, vol. 17, n 7, p.1117-1136. doi:10.1177/1461444814521595

Broqua Christophe. (2018). La " communauté homosexuelle » comme peuple transnational : Une fiction politique. L'Homme \& la Société, vol. 208, n 3, p. 143-167. doi:10.3917/lhs.208.0143.

Bryant Kelly M, Soria, Krista M. (2015). College students' sexual orientation, gender identity, and participation in Study Abroad. Frontiers: The interdisciplinary journal of study abroad, vol. 25, p. 91-106.

Cascalheira Cory, Smith Brandt. (2019). Hierarchy of desire: partner preferences and social identities of men who have sex with men on geosocial networks. Sexuality \& Culture, vol. 24, n³, pp.630-648. https://doi.org/10.1007/s12119-019-09653-z

Collins Rodney. (2012). Efféminés, Gigolos, and MSMs in the Cyber-Networks, Coffeehouses, and 'Secret Gardens' of Contemporary Tunis. Journal of Middle East Women's Studies, vol. 8, n 3, p. 89-112.

Develotte Christine. (2006). Décrire l'espace d'exposition discursive dans un campus numérique. Le Français dans le monde. Recherches et applications, p. 88-100.

Diminescu Dana. (2008). The connected migrant: an epistemological manifesto. Social Science Information, vol. 47, $\mathrm{n}^{\circ}$ 4, p. 565-579. https://doi.org/10.1177/0539018408096447

Diminescu Dana, Loveluck Benjamin. (2014). Traces of dispersion. Online media and diasporic identities. Crossings: Journal of Migration \& Culture, vol. 5, $\mathrm{n}^{\circ}$ 1, p. 23-39.

Duff Patricia. (2008). Case Study Research in Applied Linguistics, New York : Routledge.

Duvoux Nicolas. (2017). Les inégalités sociales, Paris : PUF.

Eid Paul. (2018). Les majorités nationales ont-elles une couleur? Réflexions sur l'utilité de la catégorie de «blanchité » pour la sociologie du racisme. Sociologie et sociétés, vol. 50, $\mathrm{n}^{\circ} 2, \quad \mathrm{p}$. 125-149. https://doi.org/10.7202/1066816ar

El-Tayeb Fatima. (2012). "Gays who cannot properly be gay": Queer Muslims in the neoliberal European city. European Journal of Women's Studies, vol. 19, n 1, p. 79-95. https://doi.org/10.1177/1350506811426388

Fox Jessie, Warber, Katie M. (2015). Queer identity management and political self-expression on social networking sites: A co-cultural approach to the spiral of silence. Journal of Communication, vol. 65, $\mathrm{n}^{\circ}$ 1, p. 79-100.

Gray John, Baynham Mike. (2020). Narratives of Queer Migration. In Hall, K. and Barrett, R. (eds.) The Oxford Handbook of Language and Sexuality. Oxford University Press. [non paginé]

Green, Adam Isaiah. (2013). "Erotic Capital and the Power of Desirability: Why "Honey Money" is a Bad Collective Strategy for Remedying Gender Inequality." Sexualities, p. 16:137-158

Guichon Nicolas. (2020). L'étudiant international : figure de l'individu mobile et connecté, Le Français dans le Monde - Recherches et applications, $\mathrm{n}^{\circ} 68$, pp. 158-169.

Guillaumin Colette. (1992). Une société en ordre. De quelques-unes des formes de l'idéologie raciste, Sociologie et sociétés, vol. 24, $\mathrm{n}^{\circ}$ 2, p. 13-23.

Hobbs Mitchell, Owen Stephen, Gerber Livia. (2017). Liquid love? Dating apps, sex, relationships and the digital transformation of intimacy. Journal of Sociology, vol. 53, $\mathrm{n}^{\circ}$ 2, p. 271-284. https://doi.org/10.1177/1440783316662718 
Hutchby Ian. (2014). Communicative affordances and participation frameworks in mediated interaction. Journal of Pragmatics, 72(C), 86-89. https://doi.org/10.1016/j.pragma.2014.08.012

Jones Rodney. (2011). Dancing, skating and sex: Action and text in the digital age. Journal of Applied Linguistics, vol. 6, n 3, p. 283-302. https://doi.org/10.1558/japl.v6i3.283

Jones Rodney. (2018). Mediated discourse analysis in language and sexuality research. In Hall, K. and Barrett, R. (eds.) The Oxford Handbook of Language and Sexuality. Oxford University Press. [non paginé]

King Russell, Raghuram, Parvati. (2013). International Student Migration: Mapping the Field and New Research Agendas. Population, Space and Place, vol. 19, n 2, p. 127-137.

Lardellier Pascal. (2012). « Le Online Dating, au coeur de la communication électronique », Netcom, vol. 26, $\mathrm{n}^{\circ} 1 \& 2$, p. 77-96. [http://journals.openedition.org/netcom/593]

Leurs Koen, Prabhakar Madhuri. (2018). Doing Digital Migration Studies: Methodological Considerations for an Emerging Research Focus. In Zapata-Barrero, Ricard, Yalaz, Evren (eds.), Qualitative Research in European Migration Studies, IMISCOE Research Series, p. 247 - 266.

Licoppe, Christian. (2016). Mobilités et rencontres dans les espaces publics urbains à l'âge des médias positionnels : Coutures, plis et rencontres avec des " inconnus pseudonymes ». Réseaux, vol. 200, $\mathrm{n}^{\circ}$ 6, p. 117-143. doi:10.3917/res.200.0117.

Licoppe Christian, Rivière, Carole Anne, Morel Julien. (2016). Grindr casual hook-ups as interactional achievements. New Media \& Society, vol. 18, n 11, p. 2540-2558. https://doi.org/10.1177/1461444815589702

Madianou Mirca, Miller Daniel. (2012). Migration and new media transnational families and polymedia. Londres : Routledge.

Mowlabocus, Sharif. (2020). A kindr Grindr. Moderating race(-ism) in technospaces of desire. Lille : Conférence plénière au colloque Mediasex.

Patterson Jeffrey, Leurs Koen. (2019). “We Live Here, and We Are Queer!” Young Adult Gay Connected Migrants' Transnational Ties and Integration in the Netherlands. Media and Communication, vol. 7, n ${ }^{\circ}$ 1, p. 90-101. https://doi.org/10.17645/mac.v7i1.1686

Paveau Marie-Anne. (2017). L'analyse du discours numérique. Paris, Hermann éditeurs.

Provencher Denis M., Peterson, David. (2020). Diasporic Sexual Citizenship: Queer Language, (Im)Possible Subjects, and Transfiliation. In Hall, K. and Barrett, R. (eds.) The Oxford Handbook of Language and Sexuality. Oxford University Press. [non paginé]

Shield Andrew. (2017). New in town: Gay immigrants and geosocial dating apps. In A. Dhoest, L. Szulc, \& B. Eeckhout (Eds.), LGBTQs, media, and culture in Europe: Situated case studies. London : Routledge, p. 244-261.

Shield Andrew. (2018). "Grindr Culture: Intersectional and Socio-Sexual." Ephemera: theory \& politics in organization, vol. 18, $\mathrm{n}^{\circ}$ 1, p. 149-161. http://search.proquest.com/docview/2059598831/

Trawalé Damien. (2016). La reproduction sans encombre des rapports de race : le cas des gays noirs. Migrations Société, vol. 163, n 1, p. 61-74. doi:10.3917/migra.163.0061.

Vörös Florian. (2018). Fantasmes de virilité, blanchité et masculinité hégémonique en contexte gai parisien. L'Homme \& la Société, vol. 208, n 3, p. 197-222. doi:10.3917/lhs.208.0197

\section{Remerciements :}

Je remercie Christelle Combe, Brahim Azaoui et Simon Collin pour leurs commentaires qui ont grandement contribué à améliorer cet article et Jean Carteron pour son aide précieuse lors du recueil. 